\title{
Horseshoe periodic orbits in the restricted three body problem
}

\author{
Jaume llibre \\ Dept. Matemàtiques. Universitat Autònoma de Barcelona, Edifici $C$ \\ 08193 Bellaterra, Barcelona, Spain.E-mail: jllibre@mat.uab.es \\ and \\ Mercè Ollé \\ Dept. de Matemàtica Aplicada I, ETSEIB (UPC), Diagonal 647, \\ 08028 Barcelona, Spain.E-mail: olle@ma1.upc.es
}

\begin{abstract}
We consider the circular restricted three-body problem (CRTBP) in the synodical system of coordinates for values of the Jacobi constant $C$ in the interval $\left(3, C_{1}\right)$ (where $C_{1}$ is the value of $C$ at the collinear equilibrium point $L_{1}$ ). We describe the existence of families of horseshoe periodic orbits when varying the mass parameter and the Jacobi constant. The relation between such orbits and the invariant manifolds of the Lyapunov families of periodic orbits around the collinear equilibrium point $L_{3}$ is also analysed.
\end{abstract}

Key Words: coorbital motion, periodic orbits, restricted three-body problem, invariant manifolds

\section{INTRODUCTION}

This paper is a natural continuation of a previous one about horseshoe periodic orbits in the CRTBP [3]. Actually the original motivation of this study was to describe the motion of Saturn coorbital satellites Janus (also called 1980S1) and Epimetheus (also 1980S3) in this simple model. More precisely, in [3] we showed the existence of new families of stable horseshoe periodic orbits (in the framework of the CRTBP) closely related to the actual motion of $1980 \mathrm{~S} 1$ and 1980S3. We described a mechanism of generation of horseshoe periodic orbits for $\mu>0$ and very small, which inherited the dynamics from the $\mu=0$ case. Such mechanism gave answer to the natural question about the origin and location of these horseshoe periodic orbits. We also carried out a numerical exploration for this partic- 
ular value of $\mu$, and we computed some families of horseshoe periodic orbits in a systematic way, for different values of $C$ in the interval $\left(2.9999, C_{1}\right)$.

Our goal in this paper is to analyse the existence of horseshoe periodic orbits in the CRTBP for any value of the mass parameter $\mu \in(0,1 / 2)$. Of course, a mechanism suitable to describe the horseshoe periodic orbits for $\mu>0$ and small does not apply for any given value of $\mu$. We will see how the invariant manifolds of the Lyapunov orbit emanating from $L_{3}$ play a key role in the mechanism that explains the existence and location of the horseshoe periodic orbits.

On the other hand, for a fixed value of $C$, we obtain a rich structure of families of horseshoe shaped orbits. In fact, the complexity of this behaviour is related to the invariant manifolds of the periodic orbits emanating, not only from $L_{3}$ but also from $L_{1}$ and $L_{2}$.

Finally, we refer the interested reader to [3] for comments on other related papers, both from analytical and numerical points of view (see references therein).

\section{THE RESTRICTED THREE-BODY PROBLEM}

We consider a system of three bodies in an inertial (called sidereal) reference system: two bodies called primaries $\left(m_{1}\right.$ and $\left.m_{2}\right)$ of masses $1-\mu$ and $\mu$ (respectively, in suitable units), describing circular orbits about their common center of mass (located at the origin of coordinates) in a plane, and a particle of infinitesimal mass which moves in the same plane under the gravitational force of the primaries but has negligible effect on their motion. The problem of describing the motion of the particle is the planar circular restricted three-body problem (CRTBP). The equations of motion in a rotating (called synodical) system of coordinates, $x$ and $y$, which rotates with the primaries [6] are

$$
\begin{aligned}
& x^{\prime \prime}-2 y^{\prime}=\frac{\partial \Omega}{\partial x}, \\
& y^{\prime \prime}+2 x^{\prime}=\frac{\partial \Omega}{\partial y},
\end{aligned}
$$

where

$$
\Omega(x, y)=\frac{1}{2}\left(x^{2}+y^{2}\right)+\frac{1-\mu}{r_{1}}+\frac{\mu}{r_{2}}+\frac{1}{2} \mu(1-\mu),
$$

and $r_{1}^{2}=(x-\mu)^{2}+y^{2}, r_{2}^{2}=(x-\mu+1)^{2}+y^{2}$ are the distances between the particle and the big and small primary respectively, and I stands for $d / d t$. It is well known that these differential equations have the so called 
Jacobi first integral

$$
x^{2}+y^{\prime 2}=2 \Omega(x, y)-C
$$

and 5 equilibrium points: the collinear points, $L_{1}, L_{2}$ and $L_{3}$ (on the left of $m_{2}$, between $m_{1}$ and $m_{2}$, and on the right of $m_{1}$ respectively), and the equilateral ones, $L_{4}$ and $L_{5}$. If one computes the value of the Jacobi constant at the equilibrium points $C_{i}=C\left(L_{i}\right)$ for any value of $\mu \in(0,1 / 2)$, one has

$$
3=C_{4}=C_{5}<C_{3}<C_{1}<C_{2},
$$

and $C_{3}=C_{1}$ for $\mu=1 / 2$.

\section{HORSESHOE PERIODIC ORBITS AND THE INVARIANT MANIFOLDS OF LYAPUNOV PERIODIC ORBITS EMANATING FROM $L_{3}$}

We call a horseshoe periodic orbit a periodic solution in which the particle (its projection on the $(x, y)$ coordinates) follows a path which surrounds only the positions of three equilibrium points $L_{3}, L_{4}$ and $L_{5}$ and has two orthogonal crossings with $y=0$; that is, for $t=0$ the initial condition of the orbit is $\left(x, 0,0, y^{\prime}\right)$, for certain $x$, and $y^{\prime}$, and for $t=T / 2, T$ being the period, the orbit is at a point $\left(\bar{x}, 0,0, \bar{y}^{\prime}\right)$ for certain $\bar{x}$, and $\bar{y}^{\prime}$. It is well known that the equations of motion of the CRTBP satisfy the symmetry

$$
\left(x, y, x^{\prime}, y^{\prime}, t\right) \rightarrow\left(x,-y,-x^{\prime}, y^{\prime},-t\right)
$$

and that any solution with two orthogonal crossings with $y=0$ (at $t=0$ and $t=T / 2$ ) becomes symmetric with respect to the $x$ axis, and periodic of period $T$. We remark that in [3] we only considered horseshoe periodic orbits with exactly two consecutive orthogonal crossings with the $x$ axis; in this paper, we allow the horsehoe periodic orbit to have several crossings with the $x$ axis; however, since we consider symmetrical orbits, they will always have exactly two orthogonal crossings with $y=0$.

From the definition given for a horseshoe periodic orbit and the knowledge of the zero velocity curves of the CRTBP [6], it is clear that the natural range of the Jacobi constant $C$ is $C<C_{1}$. In fact, we will restrict our study to the interval $3<C<C_{1}$. On the other hand, the linearized vector field at each collinear point $L_{i}, i=1,2,3$, exhibits a behaviour of saddle-center type (that is, the characteristic exponents are $\pm \lambda \in R$, and $\pm i b \in C, b \in R$ ), and the infinitesimal oscillations around $L_{i}$ (associated with the exponents $\pm i b$ ) for $C<C_{i}$, continue along a family of plane retrograde periodic orbits surrounding $L_{i}$, the so called family of Lyapunov periodic orbits [6]. 

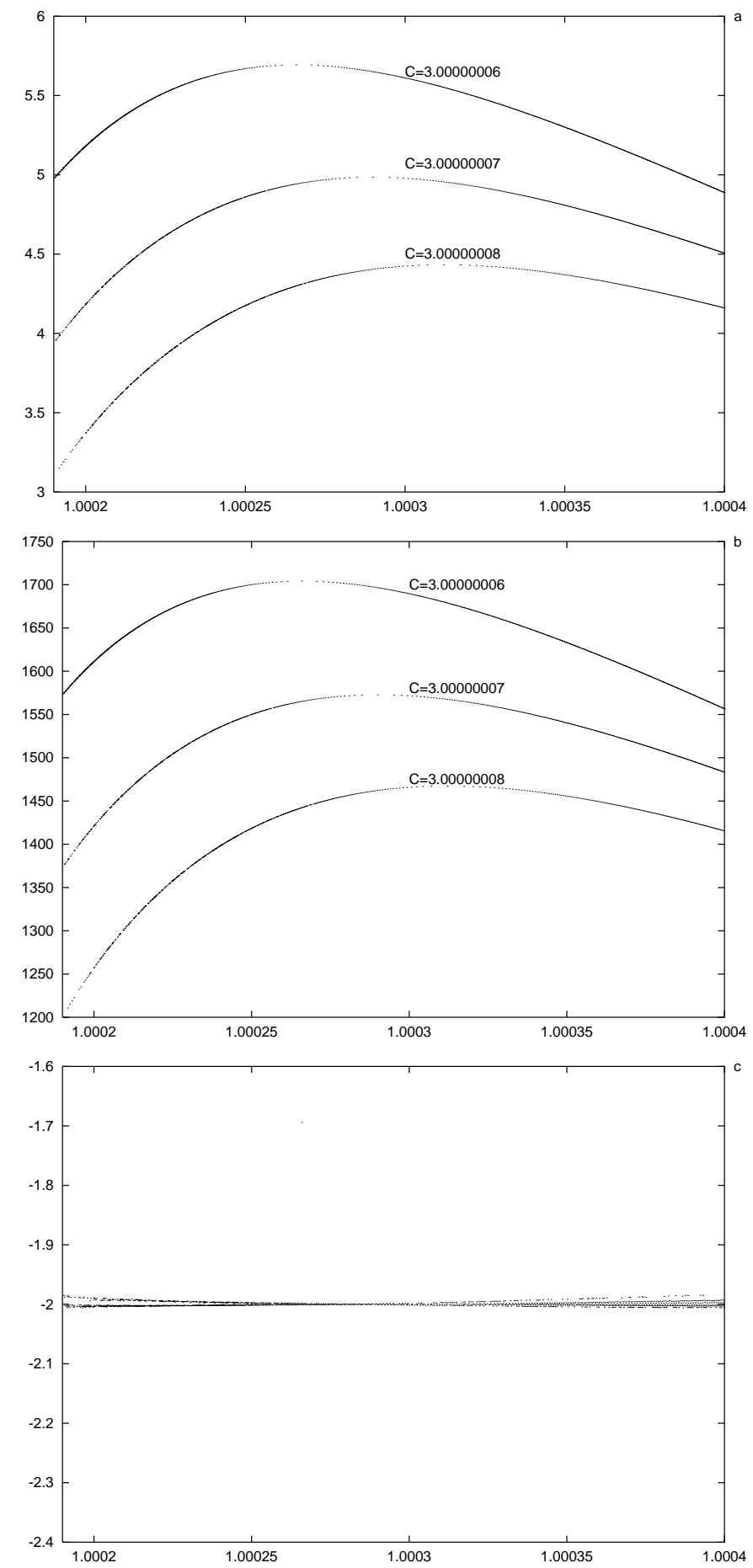

FIG. 1. Initial conditions $x$ versus the minimum angular separation $\theta_{\min }$ of families of horseshoe periodic orbits for different values of $C \in\left(C_{3}, C_{1}\right)$ (Fig. 1 a). For every horseshoe periodic orbit computed, we plot the value of the half period $T / 2$, in days, (Fig. 1 b) and the stability parameter (Fig. 1 c). 

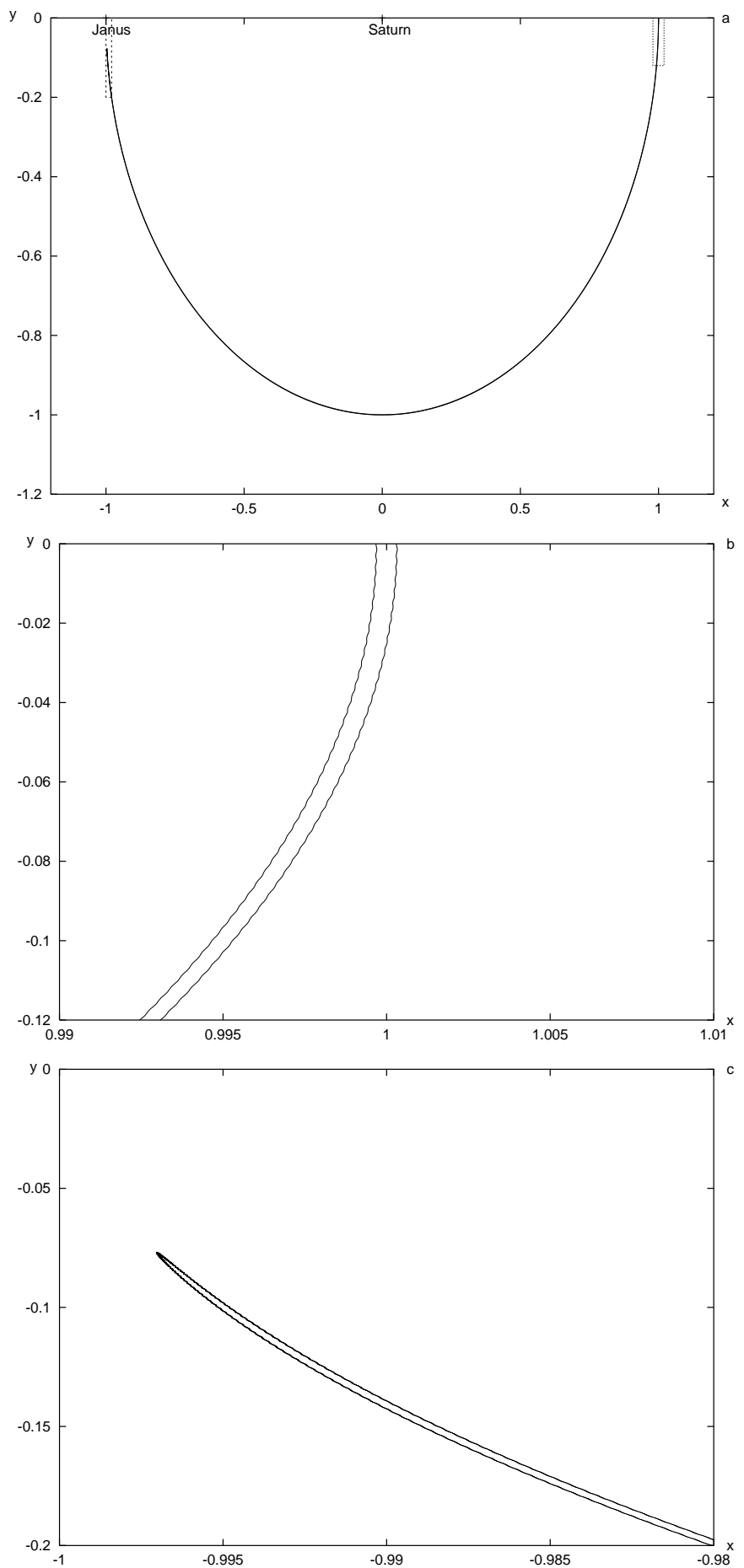

FIG. 2. a. A horseshoe periodic orbit suitable for Janus and Epimetheus. b. Detail. c. The effect of return when both satellites approach each other. 
Since the collinear points $L_{1}, L_{2}$ and $L_{3}$ are of saddle-center type, the same occurs for the Lyapunov periodic orbits around and close to them. Thus, for a given Lyapunov orbit emanating from $L_{i}, i=1,2,3$, denoted by $L O\left(L_{i}\right)$, we can consider the two branches of the stable invariant manifolds $W_{L O\left(L_{i}\right)}^{s, 1}, W_{L O\left(L_{i}\right)}^{s, 2}$; and the unstable ones, $W_{L O\left(L_{i}\right)}^{u, 1}, W_{L O\left(L_{i}\right)}^{u, 2}$. These stable (unstable) manifolds are topological cylinders, foliated by orbits tending to $L O\left(L_{i}\right)$ for forward (backward) time. The first, or j-th in general, intersection between these cylinders and the $y=0$ hyperplane are, in general, topological circles. We denote by $\gamma_{j}^{s, i}$ and $\gamma_{j}^{u, i}$, for $i=1,2$, such circles in the plane $\left(x, x^{\prime}\right)$.

In order to compute these invariant manifolds, we fix $\mu>0$ and $C<$ $C_{3}$, so $L O\left(L_{3}\right)$ is determined, and we follow only the manifolds $W_{L O\left(L_{3}\right)}^{s, 1}$ and $W_{L O\left(L_{3}\right)}^{s, 2}$, since $W_{L O\left(L_{3}\right)}^{u, 1}$ and $W_{L O\left(L_{3}\right)}^{u, 2}$ are obtained from them using the above symmetry of the equations. We can proceed to the numerical globalization of an invariant manifold by means of the monodromy matrix (for each point of the $L O$ we follow the solution tangent to the eigenvector of the monodromy matrix with eigenvalue $\lambda<1$ for backward time), or through a similar process using the Poincaré map and its differential (see [5] for the Hill's problem).

Concerning the existence of horseshoe periodic orbits, we know from [3] that there exist horseshoe periodic orbits for $\mu>0$ and very small. We show, as an interesting example, some families of horseshoe periodic orbits for $\mu=3.5 * 10^{-9}$, which is the value of the mass parameter if we consider Saturn and the coorbital satellites Janus and Epimetheus in the framework of the CRTBP; that is, Saturn as the big primary, Janus as the small one and Epimetheus as an infinitesimal body. On one hand, from the orbital elements of the satellites, we take into account that Epimetheus describes, in this simplified model, a horseshoe periodic orbit of period 2928.6 days, the minimum angular separation between both satellites is 5.6 degrees and the two orthogonal crossings with $y=0$ and $x>0$ correspond to the values of $x=1.00033$ and $x=0.999669$. On the other hand, we show the families obtained for $\mu=3.5 \cdot 10^{-9}$ and different values of $C \in\left(C_{3}, C_{1}\right)$ in Figure 1; we plot the initial value $x$, the half-period (in days), the angle of separation, in the $(x, y)$ projection, between the small primary and the infinitesimal body at the return (closest) point in its horseshoe periodic orbit and the stability parameter of the periodic orbit (defined by $\alpha=2-\operatorname{tr} M$, where $M$ is the monodromy matrix for each periodic orbit computed). We also plot a particular horseshoe periodic orbit in Figure 2, suitable to describe the motion of Saturn coorbital satellites (see the details in [3]).

We remark at this point that, according to [1] and [4], since these periodic orbits do not have a period multiple of $2 \pi$, they can be continued as families of periodic orbits for the planar three-body problem when the mass of the 
third body is small enough. Therefore, this result allows us to derive the existence of horseshoe motion in the three body problem, with masses near the masses of Saturn and Janus as before, and Epimetheus with a mass (in the units that we are using) equal to $1.5 * 10^{-9}$.

But, as we mentioned in the introduction, we are interested in horseshoe periodic orbits in the CRTBP for any value of the mass parameter $\mu \epsilon$ $(0,1 / 2]$. We describe now the relation between the existence of horseshoe periodic orbits and the shape of the invariant manifolds of the periodic orbits around $L_{3}$, when increasing the mass parameter. To do so, we have computed, for different fixed values of $\mu>0$, the corresponding Lyapunov orbit $L O\left(L_{3}\right)$, for $C<C_{3}$, its invariant manifolds as well as the family of horseshoe periodic orbits. The numerical exploration carried out shows that, given a value of $\mu>0$, we can expect to have horseshoe periodic orbits if the flow (partially or totally) on the invariant manifolds of $L O\left(L_{3}\right)$ has also a horseshoe shape.

More precisely, our results show three kind of phenomena according to the value of $\mu$ :

(i) For $0<\mu$ small and $C<C_{3}$ close to $C_{3}$, the flow on the invariant manifolds has a horseshoe shape and the curves $\gamma_{1}^{s, i}, i=1,2$, are defined for $x>0$ and give rise to homoclinic orbits. For example, we consider the Sun (S) and Jupiter $(\mathrm{J})$ as primaries, that is $\mu=\mu_{S J}=0.000953875$, and $C=3.001906457<C_{3}$, we have computed the $L O\left(L_{3}\right)$ and the global manifolds $W_{L O\left(L_{3}\right)}^{s, 1}, W_{L O\left(L_{3}\right)}^{s, 2}$ until the first intersection with $y=0$ takes place. We plot them in Figure 3. We remark, first of all, that we obtain broken curves instead of a curve homeomorphic to $S^{1}$ (see Figure $3 \mathrm{c}$ and f). These discontinuities in the $(x, \dot{x})$ plane take place due to the loops that some orbits on $W_{L O\left(L_{3}\right)}^{s, i}, i=1,2$, describe when they are projected in the $(x, y)$ plane (see Figure 4$)$; that is, the loops are responsible for displaying changes in the number of cuts with $y=0$, and therefore discontinuities in the $(x, \dot{x})$ projection (see also [2]).

However, each point obtained from the intersection between $\gamma_{1}^{s, i}, i=1,2$, and the $x^{\prime}=0$ axis, gives a transversal symmetric homoclinic orbit which tends asymptotically, in forward and backward time, to the Lyapunov orbit $L O\left(L_{3}\right)$. Therefore, we could introduce the Bernoulli shift for each intersection and derive the existence of infinitely many families of periodic orbits, which in particular will contain the families of horseshoe periodic orbits.

Now, we decrease just a little bit the Jacobi constant $C$ and we repeat the computations. For instance, for $C=3.001902309$, we plot the curves $\gamma_{1}^{s, i}, i=1,2$, in the $\left(x, x^{\prime}\right)$ plane in Figure 5 . As we can see, there is a rather rich and intricate structure of families of homoclinic orbits, very close ones to the others, and, at the same time, each one implies the existence of infinitely many families of periodic orbits. 

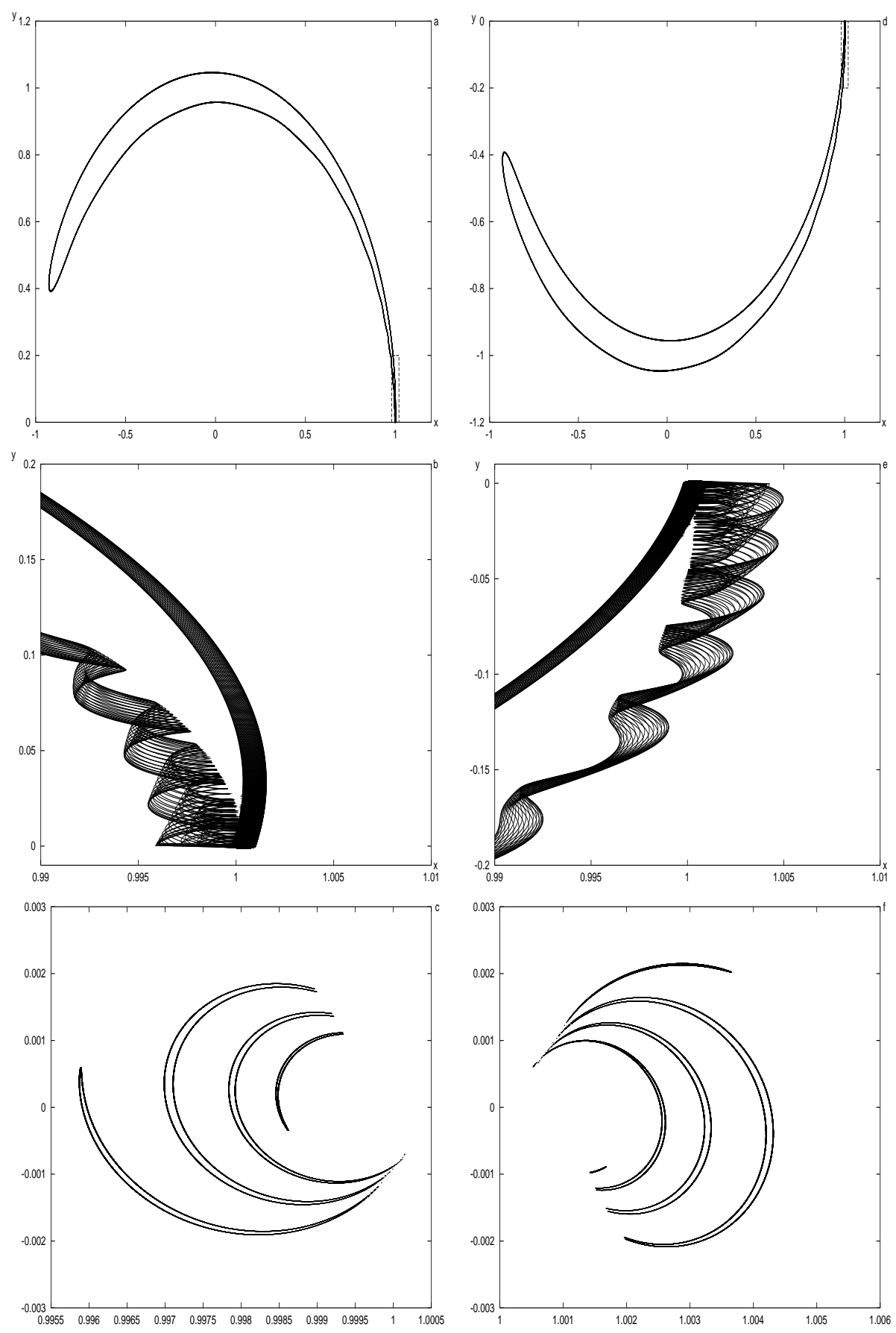

FIG. 3. $\mu=\mu_{S J}, C=3.001906457$. a. The flow on $W_{L O\left(L_{3}\right)}^{s, 1}$. b. Detail. c. The curve $\gamma_{1}^{s, 1}$ in the $(x, \dot{x})$ plane. d. The flow on $W_{L O\left(L_{3}\right)}^{s, 2}$. e. Detail. f. The curve $\gamma_{1}^{s, 2}$ in the $(x, \dot{x})$ plane. 


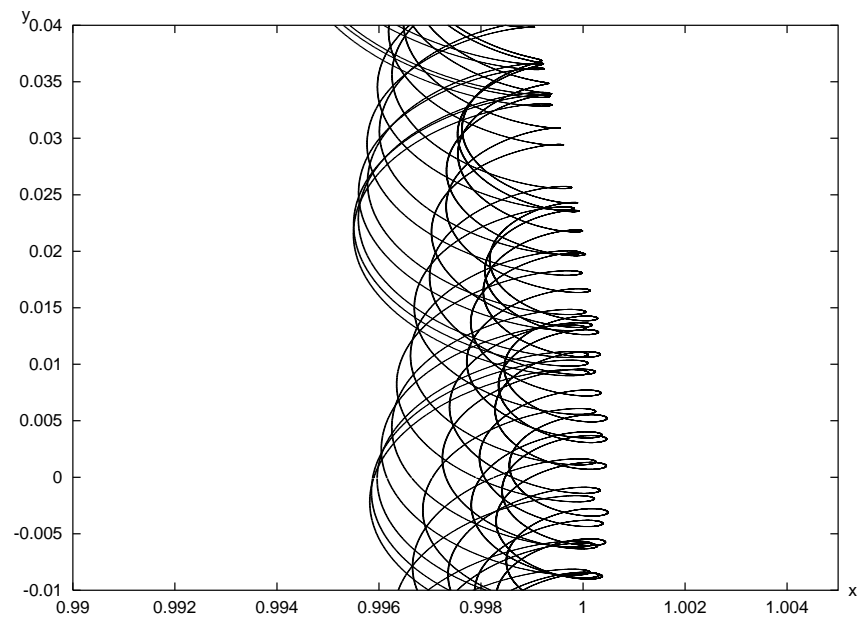

FIG. 4. We plot some orbits on $W_{L O\left(L_{3}\right)}^{s, 1}$ (projection on the $(x, y)$ plane). We can see the loops responsible for the discontinuities in the $(x, \dot{x})$ projection when the section $y=0$ is considered.

In order to understand this behaviour, we plot some orbits of the flow on the stable invariant manifold; as mentioned above, when only the $(x, y)$ projection is considered, each solution 'leaves' the Lyapunov periodic orbit for backward time, describes a horseshoe shape (we will say a horseshoe path) and we follow the solution until the intersection with the section $y=0$ takes place (actually it passes close the Lyapunov orbit again). We plot eight chosen orbits on $W_{L O\left(L_{3}\right)}^{s, 1}$ in Figure 6: we only plot the $(x, y)$ projection described by the orbits when they have already described the horseshoe path and go back to the $y=0$ section. As we can see in Figure 6, comparing it with Figure 4, there are some orbits (Figures 6 a, b and c) which after the horseshoe path, they do not intersect the $y=0$ section (as can be shown in Figure 4), but they go upwards again describing another horseshoe path and finally they intersect the $y=0$ section. On the other hand, they also describe a loop close to the $y=0$ section. This behaviour shown only for some selected orbits of the flow on the stable invariant manifold, takes place also for many other orbits of the flow on $W^{s, 1}$; therefore we obtain Figure 5 a (Figure $5 \mathbf{b}$ ) when the curve $\gamma_{1}^{s, 1}$ $\left(\gamma_{1}^{s, 2}\right)$ is considered.

On the other hand, for different values of $\mu$, we also show the relation between the horseshoe periodic orbits and the horseshoe shape of the invariant manifolds of the periodic orbits emanating from $L O\left(L_{3}\right)$. We plot in Figure 7, for example for $\mu=0.0089$ and $C=3.017$, the invariant manifolds $W_{L O\left(L_{3}\right)}^{s, 1}, W_{L O\left(L_{3}\right)}^{u, 2}, W_{L O\left(L_{3}\right)}^{s, 2}$ and three horseshoe periodic orbits: the 

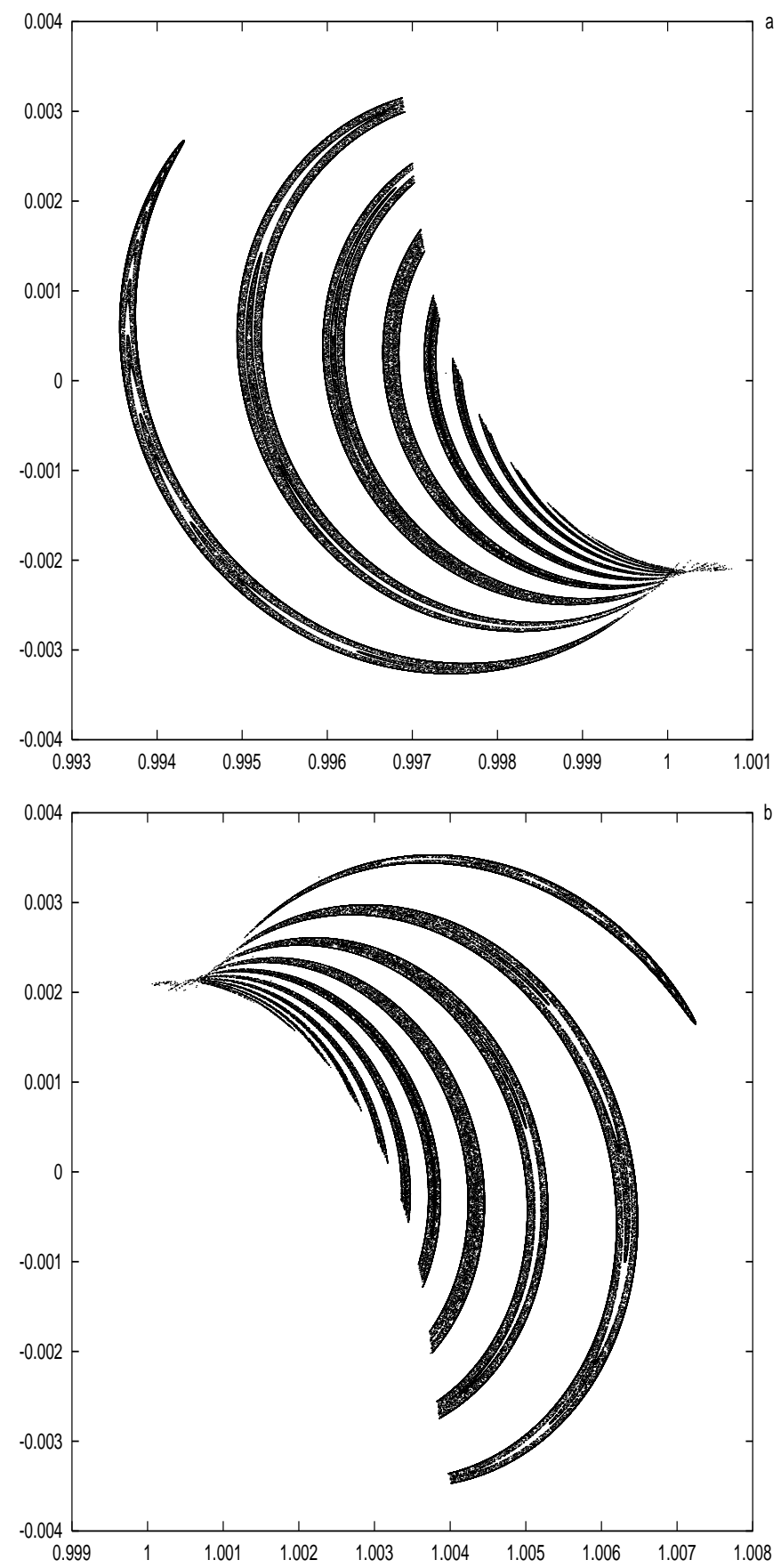

FIG. 5. $\mu=\mu_{S J}, C=3.001902309$. a. The curve $\gamma_{1}^{s, 1}$ in the $(x, \dot{x})$ plane. b. The curve $\gamma_{1}^{s, 2}$ in the $(x, \dot{x})$ plane. 

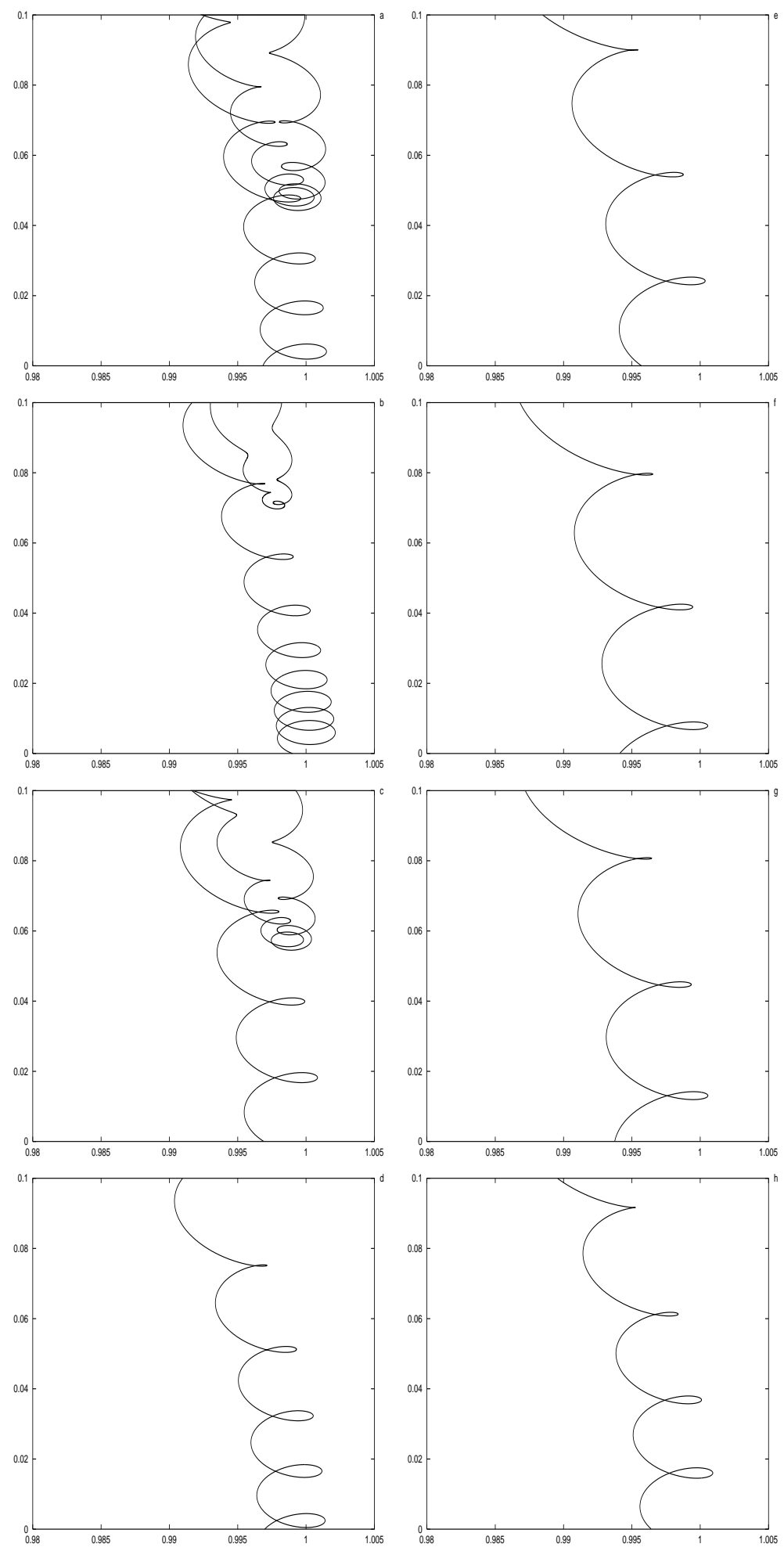

FIG. 6. $\mu=\mu_{S J}, C=3.001902309$. We plot the $(x, y)$ projection of eight chosen orbits of the flow on $W_{L O\left(L_{3}\right)}^{s, 1}$. Some orbits have two horseshoe paths before they intersect the $y=0$ section (Fig. $6 \mathbf{a}, \mathbf{b}$ and $\mathbf{c}$ ) and some have only one (Fig. $6 \mathbf{d}, \mathbf{e}, \mathbf{f}$, $\mathbf{g}$ and $\mathbf{h})$. We can also see the loop of each orbit close to this intersection. 

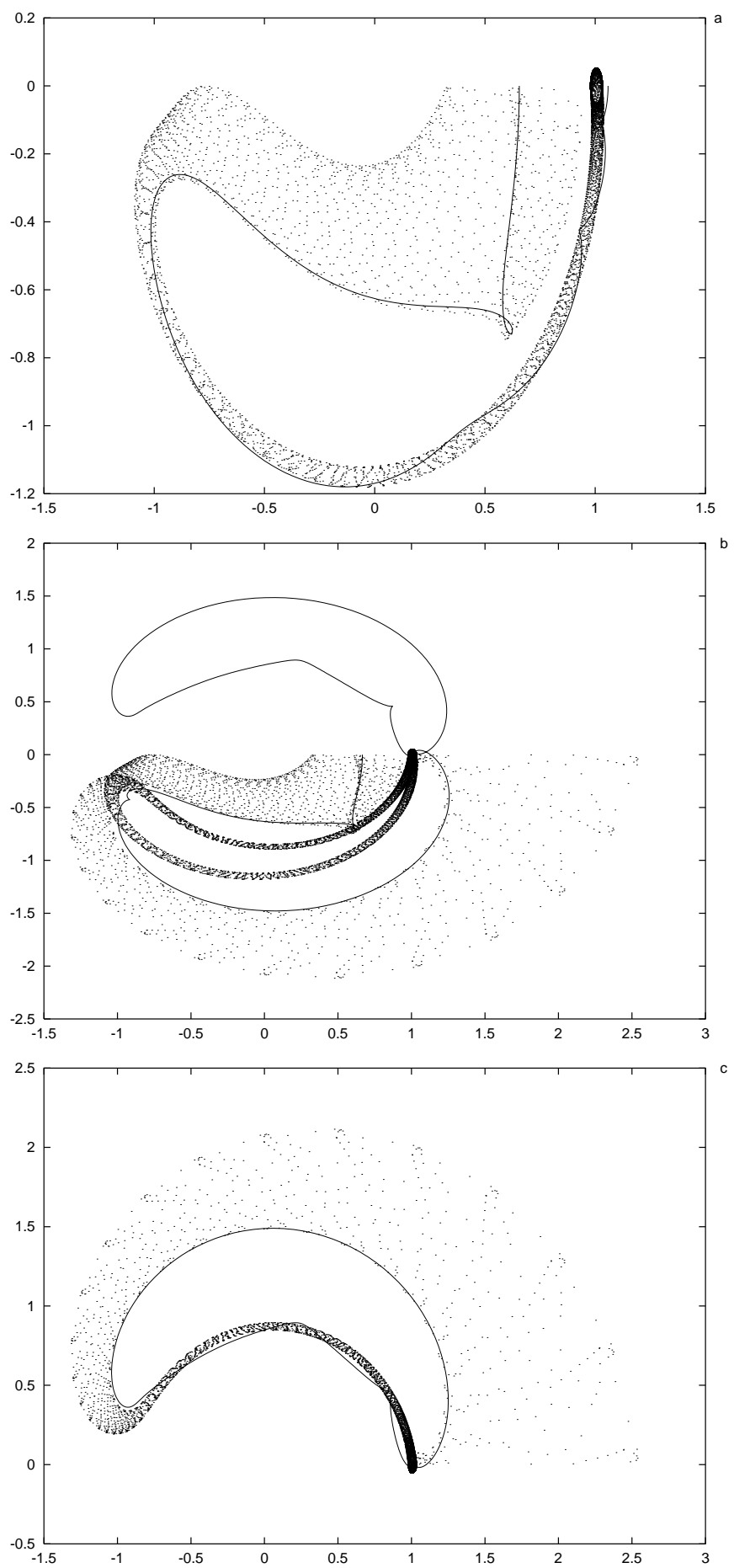

FIG. 7. Horseshoe periodic orbits $-(x, y)$ projection- for $\mu=0.0089$ and $C=3.017$ and the invariant manifolds of the corresponding Lyapunov orbit around $L_{3}$. 

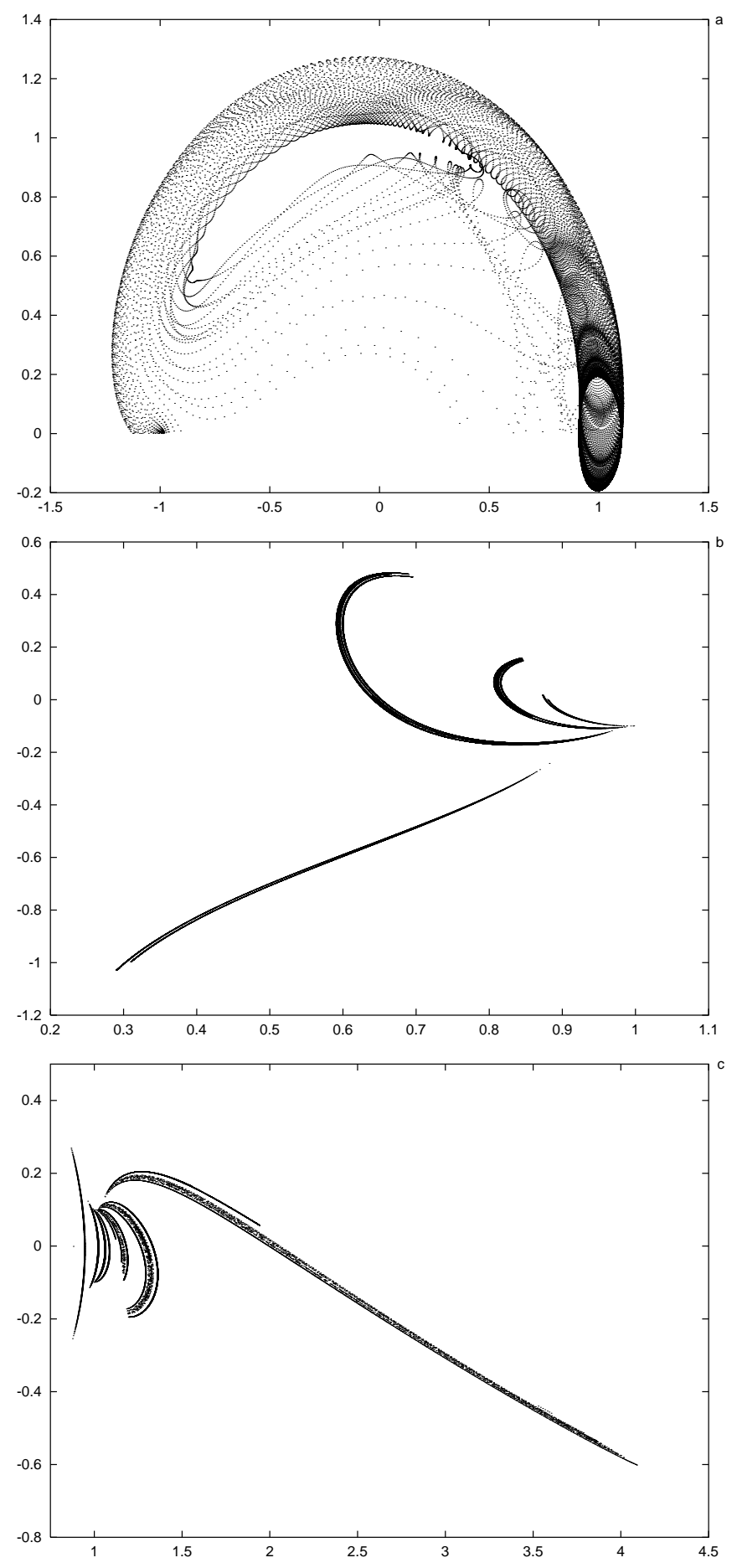

FIG. 8. $\mu=0.01, C=3.01$. From top to bottom: a. The flow on $W_{L O\left(L_{3}\right)}^{s, 1}$ in the $(x, y)$ plane. b. The curve $\gamma_{1}^{s, 1}$ in the $(x, \dot{x})$ plane. c. The curve, in the $(x, \dot{x})$ plane, obtained from the second intersection between $W_{L O\left(L_{3}\right)}^{s, 1}$ and $y=0, x>0$. 


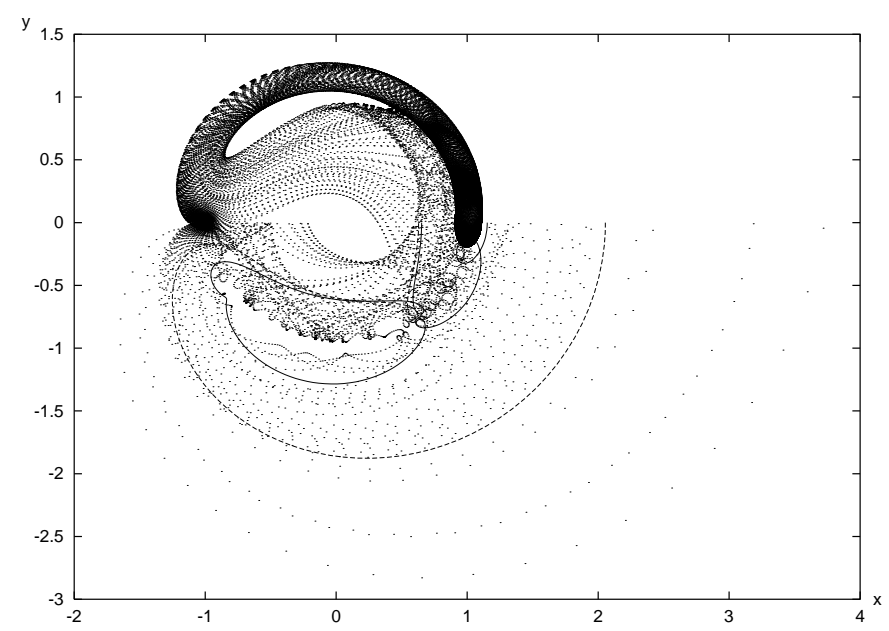

FIG. 9. Two horseshoe periodic orbits for $\mu=0.01$ and $C=3.01$ and the invariant manifold $W_{L O\left(L_{3}\right)}^{s, 1}$ of the corresponding Lyapunov orbit around $L_{3}$, until the second intersection with $y=0$ is achieved.

first one with only two (orthogonal) crossings with the $x$ axis, the second one with six crossings, and the third one with four crossings; we can see how the horseshoe shape of the periodic orbits is closely related to the flow of the manifold.

(ii) As far as $\mu$ increases, the domain of attraction of the small primary $m_{2}$ is more powerful, and the flow on the invariant manifolds of the Lyapunov family of periodic orbits (emanating from $L_{3}$ ) may pass very close to it and even collide with it, although some orbits on the invariant manifolds keep a horseshoe shape. We show, for example, in Figure 8 the flow on $W_{L O\left(L_{3}\right)}^{s, 1}$, for $\mu=0.01$ and $C=3.01<C_{3}$; although there are some orbits belonging to $W_{L O\left(L_{3}\right)}^{s, 1}$ which go to (or close to) collision with $m_{2}$, there remain other that have a horseshoe shape and there are also homoclinic orbits obtained from the curves $\gamma_{1}^{s, 1}$ at the first intersection with $y=0, x>0$ (see Figure $8 \mathbf{b}$ ) or even at the second one with $y=0, x>0$ (see Figure 8 c). Such homoclinic orbits allow the existence of horseshoe periodic orbits with an initial $x$ value far from the zero velocity curve, that is, we obtain within the same family of horseshoe periodic orbits, for $C$ fixed, some orbits which are far from each other. In Figure 9 we display two horseshoe periodic orbits of the family obtained for $\mu=0.01$ and $C=3.01$, with initial conditions $x=1.15350741073$ and $x=2.05523296943$, and the corresponding negative value of $y^{\prime}$ obtained from the Jacobi constant.

(iii) Finally for $\mu \geq 0.1$ and $3 \leq C<C_{1}$, we could not compute any horseshoe periodic orbits since the invariant manifolds of the Lyapunov 

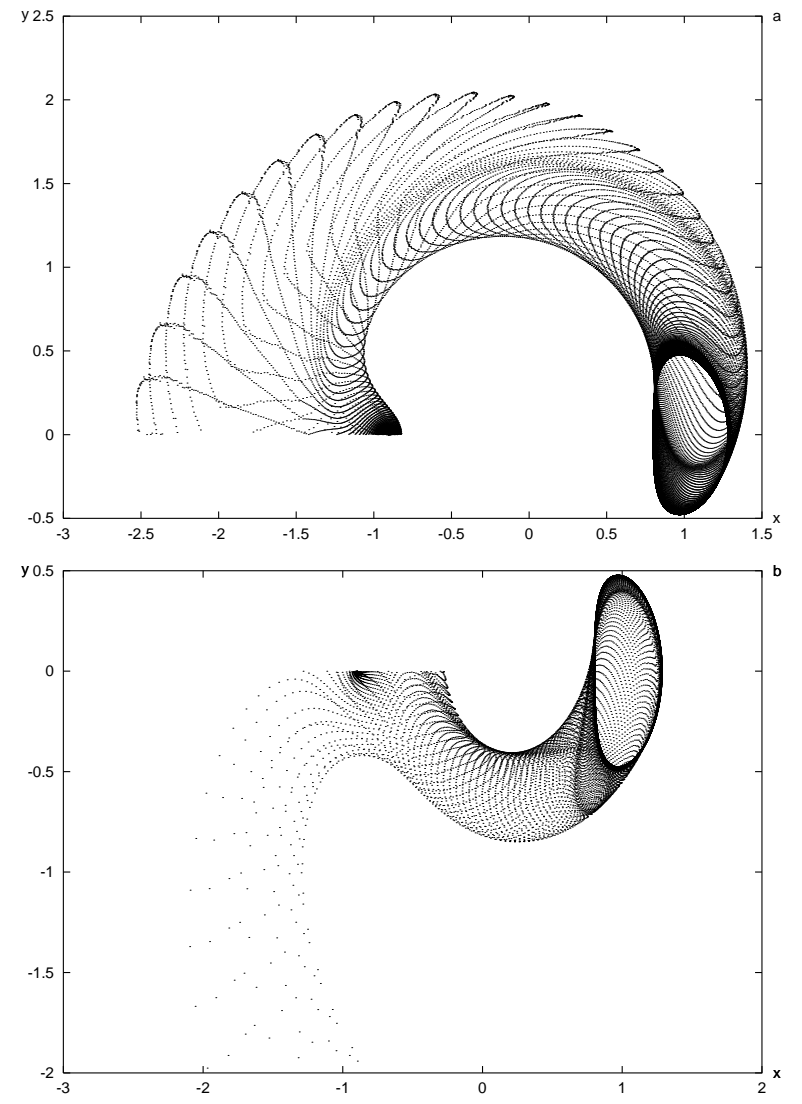

FIG. 10. $\mu=0.1, C=3.1<C_{3}$. From top to bottom: a. The flow on $W_{L O\left(L_{3}\right)}^{s, 1}$. b. The flow on $W_{L O\left(L_{3}\right)}^{s, 2}$. 
orbits emanating from $L_{3}$ do not describe a horseshoe shape anymore; see for example in Figure 10 the flow on the invariant manifolds of the corresponding Lyapunov orbit, for $C=3.1<C_{3}$ and $\mu=0.1$.

Remark 1. We have analyzed the horseshoe periodic orbits for $C<C_{3}$; of course, for $\mu$ fixed and for any fixed value of $C, C \in\left[3, C_{1}\right)$, there still exist families of horseshoe periodic orbits although the number of them is drastically reduced when $C$ increases.

Remark 2. Another interesting comment is to see the evolution, for $\mu$ and $C$ fixed, of the horseshoe periodic orbits in the same family and how the invariant manifolds of the Lyapunov orbits around $L_{1}$ and $L_{2}$ play a role in the dynamics of such orbits. See details of both remarks in [3].

\section{ACKNOWLEDGMENT}

The first author is partially supported by DGES grant number PB96-1153 and by CIRIT grant number 1999SGR-00349.

The second author acknowledges the Departament de Matemàtiques (Universitat Autònoma de Barcelona) and the Centre de Recerca Matemàtica (Bellaterra) where this research has been carried out, and is partially supported by the Catalan grant CIRIT number 2000SGR-00027 and by the Spanish grant number BFM2000-0623.

\section{REFERENCES}

1. J. D. HADJIDEMETRIOU, The continuation of periodic orbits from the restricted to the general three-body problem, Cel. Mech. 12 (1975), 155-174.

2. J. Llibre, R. Martinez ANd C. Simo, Transversality of the Invariant Manifolds Associated to the Lyapunov Family of Periodic Orbits near $L_{2}$ in the Restricted Three-Body Problem, J. Diff. Eqs. 58 (1985), 104-156.

3. J. LlibRe AND M. OllÉ, The motion of Saturn coorbital satellites in the Restricted Three-Body Problem, to appear in Astron. Astrophys., 2001.

4. K. R. Meyer And D. S. Schmidt, From the restricted to the full three-body problem, Trans. Amer. Math. Soc. 352 (2000), 2283-2299.

5. C. Simó And T. Stuchi, Central Stable/Unstable Manifolds and the destruction of KAM tori in the planar Hill problem, Cel. Mech. and Dyn. Astron. 66 (1999), $500-513$.

6. V. Szebehely, Theory of orbits, Academic Press, 1967, ch. 5. 